\title{
Designing Origami-adapted Deployable Modules for Soft Continuum Arms ${ }^{\star}$
}

\author{
Ketao Zhang and Kaspar Althoefer \\ Queen Mary University of London, Mile End, London E1 4NS, UK \\ ketao.zhangeqmul.ac.uk, k.althoefer@gmul.ac.uk
}

\begin{abstract}
Origami has several attractive attributes including deployability and portability which have been extensively adapted in designs of robotic devices. Drawing inspiration from foldable origami structures, this paper presents an engineering design process for fast making deployable modules of soft continuum arms. The process is illustrated with an example which adapts a modified accordion fold pattern to a lightweight deployable module. Kinematic models of the four-sided Accordion fold pattern is explored in terms of mechanism theory. Taking account of both the kinematic model and the materials selection, a 2D flat sheet model of the four-sided Accordion fold pattern is obtained for 3D printing. Following the design process, the deployable module is then fabricated by laminating 3D printed origami skeleton and flexible thermoplastic polyurethane (TPU) coated fabric. Preliminary tests of the prototype shown that the folding motion are enabled mainly by the flexible fabric between the gaps of thick panels of the origami skeleton and matches the kinematic analysis. The proposed approach has advantages of quick scaling dimensions, cost effective and fast fabricating thus allowing adaptive design according to specific demands of various tasks.
\end{abstract}

Keywords: Origami-folding $\cdot 3 \mathrm{D}$ printing $\cdot$ Deployable module.

\section{Introduction}

Origami folding and sculpting techniques transform flat sheet materials such as paper and cardboard into three-dimensional objects following basic and compound folds [1]. With unravelled mathematical and geometrical principles in the attractive art of paper folding [2], artistic origami folding has been sources of inspiration for engineers making innovative machines towards a wide range of engineering applications, where foldability, deployability and portability are sought after. For instance, origami folding are widely adapted in the design of versatile gift boxes in the packaging industry [3]. Taking origami creases as compliant hinges, novel compliant mechanical systems were successfully developed $[4,5]$. Origami patterns were also adapted in designing thinwalled structures [6,7], where origami pattern guides deformation and allows energy absorption in crushing process.

\footnotetext{
* This work was partially supported by research awards from the Engineering and Physical Sciences Research Council (EPSRC) under grant agreements EP/R02572X/1.
} 
By integrating various actuation technologies [8-10], passive origami structures have been adapted to active robotic systems, such as the crawling robot that folds itself from a flat sheet with embedded electronics [11], the self-foldable 3D robot functional for working and swimming [12], robot end-effector for medical devices [13] and selflocking robotic arm that can access confined spaces [14]. Of all these examples, the origami structures have been used as skeletons in place of the conventional mechanical systems composed of rigid links and kinematic pairs.

With recent advancements in materials particularly functional materials and soft filaments suitable with rapid additive manufacturing technologies, origami folds and structures have been adapted to designs of soft robots composed of compliant materials in parallel to the development of robotic systems employing rigid-foldable origami. Drawing inspiration from origami, a design method were developed for building multimaterial based machines and robots [15]. Using 3D printable soft materials (PolyJet ${ }^{\mathrm{TM}}$ ), a modular origami twisted tower was converted into a 3D printable model, leading to 3D printed semi-soft segments of a robotic manipulator [16]. In a similar way, a soft pneumatic actuator capable of extension and contraction was developed using elastomeric composite with embedded flexible sheets [8]. Most recently, soft and lightweight polymeric films including thermoplastic polyurethane (TPU) and polyethylene (PE) are used as air proof membrane of origami folding inspired soft machines. For example, a design and fabrication method was introduced to make low-cost fluid-driven artificial muscles which employ folded origami structure as skeleton and TPU film as skin [17]. A pneumatic soft robotic arm capable of a large extension and contraction ratio was developed by adapting a quadrilateral origami structure in the design of 3D printable origami exoskeletons of a pneumatic robot arm [18].

In this work, we propose an engineering design process for developing deployable modules of soft continuum arms by adapting foldable origami structures. A modified four-sided accordion fold pattern is taken as an example to demonstrate the presented process and a prototype is fabricated by laminating 3D printed TPU origami skeleton and TPU fabric skin as proof of concept of the deploayable module. The contribution of this work includes 1) engineering design process for origami-folding based deployable modules of soft continuum arms, and 2) a four-sided Accordion fold patterns based deployable module for soft continuum arm.

In the following sections, we first introduce the engineering design process for origami folding inspired deployable modules. Following this process, section III presents the kinematic modelling and 3D printable design of a modified four-sided accordion fold pattern. Section IV details the steps of fabricating the deployable module with 3D printed thick-panel origami skeleton. The paper is then concluded in Section V.

\section{The Engineering Design Process of Origami-inspired Deployable Modules for Soft Continuum Arms}

In the past decade, novel design and fabrication technologies of fully-integrated robotic mechanisms have been extensively investigated. Among the emerging technologies for fabricating multi-part and multi-material systems, the origami inspired techniques shed bright light, especially the design methodology and manufacturing process for con- 
structing three-dimensional active structure from two-dimensional flat sheet model. In contrast to the traditionally multi-body systems with disjoint components, the origamienabled mechanical systems have distinct deployability and foldability which are expected in various practical applications. In this section, we introduce an engineering design process for developing foldable origami structure based deployable modules of soft continuum arms by fusing geometric modelling, materials selection and additive manufacturing (Fig. 1).

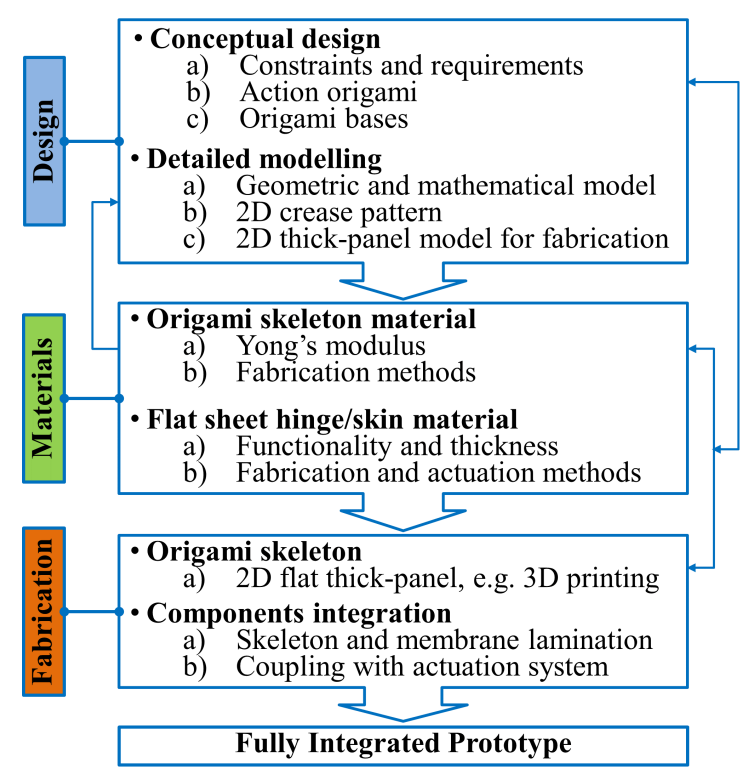

Fig. 1. The process for designing and fabricating foldable origami structure based deployable modules of soft continuum arms with multiple modules.

The design process starts from the conceptual design by defining the constraints and requirements for making an extensible continuum arm. The deployability requires the robot arm to be able to contract into a compact configuration thus adapt to confined space and to elongate in the axial direction thus enable an extended working space. A number of 3D origami structures, including the accordion [19,20], Kresling [19] and Tachi-Miura polyhedron [21], are foldable and match the requirements of extensible continuum arm. With consideration of the materials selection and possible fabrication techniques simultaneously, desired action origami are then selected from the foldable 3D origami structures.

With selected action origami in the conceptual design stage, the origami bases and their corresponding equivalent mechanisms are then extracted for exploiting the geometric and kinematic model in terms of theories of mechanisms. Based on the modelling, geometric parameters of the 2D crease pattern as well as the pre-fabrication flat 
sheet model are then defined with consideration of thickness of both the origami skeleton panels and thin sheet membranes Fig. 1.

The materials selection is a step bridging the design and fabrication steps. The material of origami skeleton is determined mainly based on the required softness (Young's Modulus in the range of $10^{5}-10^{6} \mathrm{~Pa}$ ) as well as available fabrication technologies. Further to the origami skeleton, the thin sheet membranes, which are used as flexible hinges or air-tight skin, are selected and in conjunction with fabrication approaches.

The final step is fabrication and integration of the components, including the arm and the actuation system. The origami skeletons are manufactured first using 3D printing and then combined with the thin sheet membrane through techniques including thermal laminating. The prototype is then completed by adding a actuation system which can be an external actuator (air pump and tendons, for example) or embedded actuators made from functional materials.

\section{Origami Models of the Four-Sided Accordion Fold Pattern}

In this section, we use the four-sided accordion fold pattern [20] as an example origami fold pattern for making a deployable module. The pattern is selected because its large ratio between the stowed and expanded configurations and square-shaped cross-section of the 3D bellow-like structure.

\subsection{Kinematic modelling of the Four-Sided Accordion Fold Pattern}

The origami accordion and it's modified version (Fig. 2) can be produced by folding a piece of thin-sheet paper with 2D crease pattern in Fig. 2.

As illustrated in Fig. 2(a), the original accordion pattern consists of identical panels that are in the shape of isosceles trapezium. The origami base of the crease pattern is a symmetric Miura-ori vertex, a degree-4 single-vertex folds where four creases intersect at a same point symmetric with the two co-linear creases. The symmetric Miura-ori vertex folds are assembled with bilateral symmetry and any two adjacent miura-ori vertex folds in a row can be considered as a rectangular tile [2]. By adding fold lines along the vertices of the original accordion pattern based on the method of including additional fold lines [22], it obtains a modified crease pattern in Fig. 2(b), leading to increased expansion of the origami model [20].

Looking closely at the crease pattern of rectangular tile with two symmetric Miuraori vertex in the accordion fold pattern, the kinematic equivalent of this crease pattern is an assembly of two spherical 4R linkages sharing a common revolute joint (Fig. 3(a)). The kinematic equivalent is a multi-loop linkage and has only one degree-of-freedom (DOF) as the two-spherical 4R linkages are rigidly combined. The multi-loop linkages in Fig. 3(a) is equivalent to a single loop 6R double-centered linkage (Fig. 3(b)). This can be proved by analyzing the geometric constraints of the two linkages. With consideration of the spherical motion at each vertex and the bilateral symmetry of the double-centered linkage, it revealed the linkage has three bifurcated motion branches with one DOF demonstrated by the distinct ways of folding [23]. 
(a)

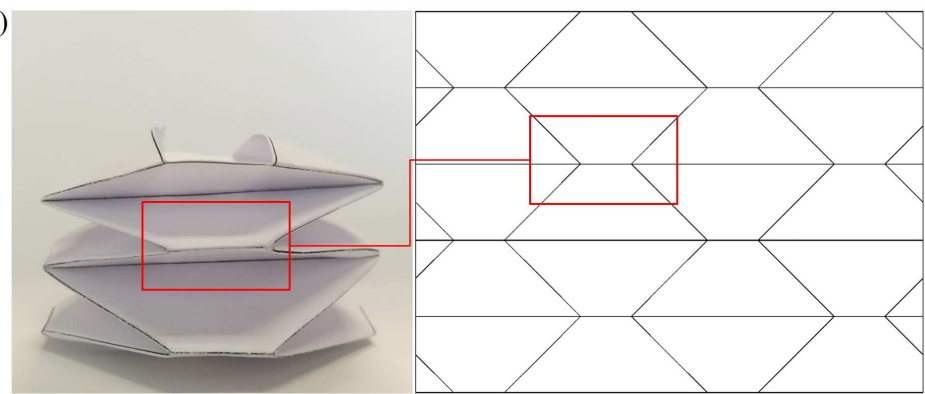

(b)

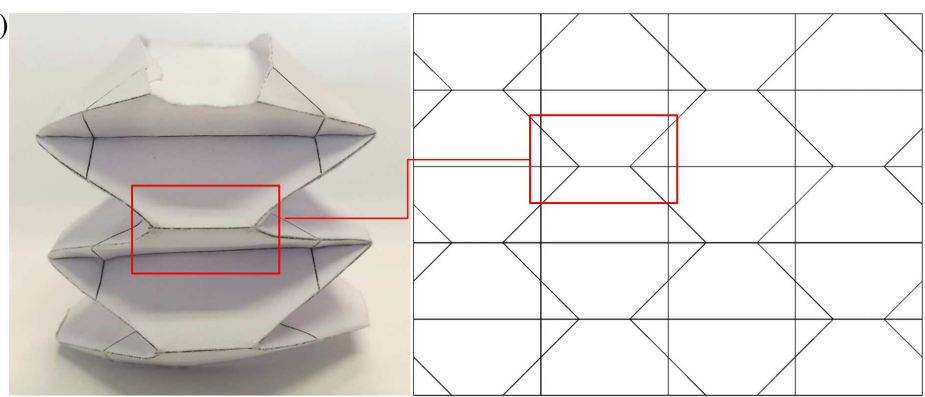

Fig. 2. The four-sided accordion fold pattern. (a) the original 2D crease pattern and 3D model, (b) the modified 2D crease pattern and corresponding 3D model.

The double-centered $6 \mathrm{R}$ linkage is a single loop mechanism that consists of six links. The product of the transform matrices is equal to the $4 \times 4$ unit matrix and the loop-closure equation [24] is expressed as

$$
\mathbf{T}_{21} \mathbf{T}_{32} \cdots \mathbf{T}_{65} \mathbf{T}_{16}=\mathbf{I}_{4}
$$

in which $\mathbf{T}_{(i+1) i}$ is the transformation matrix between $i$ th and $(i+1)$ th coordinate frames.

Considering the bilateral symmetry and shared joint between two spherical centers $O_{1}$ and $O_{2}$, the relationship of the dihedral angles are given by

$$
\begin{aligned}
& \varphi_{11}=\varphi_{13}, \varphi_{12}=\varphi_{14} \\
& \varphi_{21}=\varphi_{23}, \varphi_{22}=\varphi_{24} \\
& \varphi_{13}=\varphi_{23}, \varphi_{14}=\varphi_{24}
\end{aligned}
$$

and

$$
\tan \frac{\varphi_{11}}{2}=\frac{1}{\cos \alpha} \tan \frac{\varphi_{12}}{2}, \tan \frac{\varphi_{21}}{2}=\frac{1}{\cos \alpha} \tan \frac{\varphi_{22}}{2}
$$

in which $\varphi_{i j}$ are the dihedral angles that describe the folding of the origami corresponding to the linkage.

The relationship shows that the symmetric double-centered 6R linkage is flat-foldable. Hence, both the original multi-loop kinematic equivalent and the rectangular tile pattern are flat-foldable. 
(a)

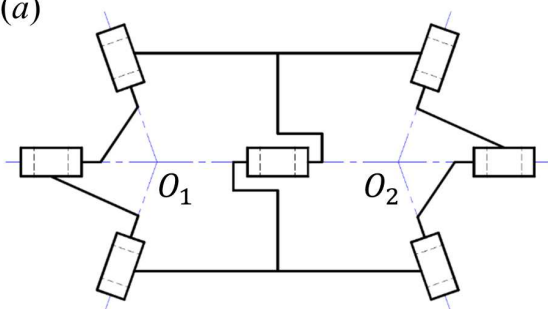

(b)

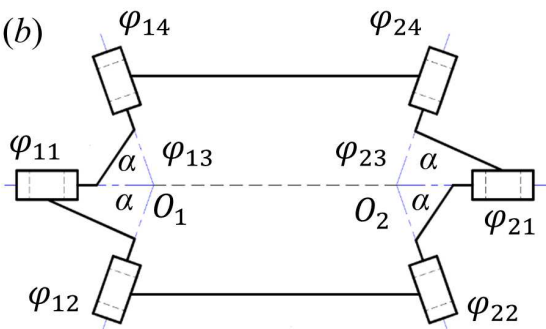

Fig. 3. Equivalent kinematic linkages of a rectangular tile crease pattern with two symmetric Miura-ori vertex folds. (a) a multi-loop linkage, (b) a 6R double-centered linkage.

\subsection{D Printable Flat Sheet Model of the Origami Skeleton}

Folding flat sheet material to a 3D origami model, the compliance of the thin sheet material allows deformation in the panels thus enable a fold pattern to move from a deployed state to a compact state even the pattern is not rigid-foldable. In contrast to the origami model made of thin sheet materials, thickness of panels of thick panel origami structures have to be accommodated to account for rigid-foldability [25].

For accommodating the thickness of panels during folding motion, both panel thickness $t_{p}$ and membrane thickness $t_{s}$ are taken into account in the design of the 3D printable flat sheet model of the origami skeleton. Following the panel-membrane model [22], the gaps corresponding to mountain and valley creases are calculated as $g_{m}=2 t_{s}$ and $g_{v}=2 t_{p}+4 t_{s}$, respectively.

(a)

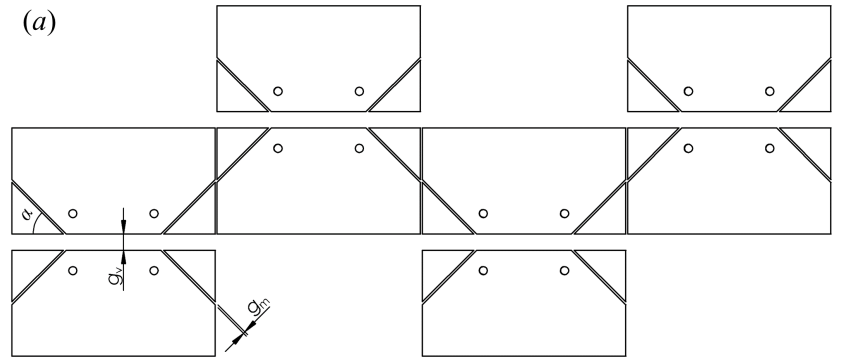

(b)

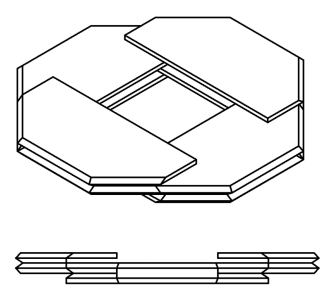

Fig. 4. The modified accordion pattern fold for a single module. (a) the model of the foldable skeleton with panel thickness $t_{p}$ and membrane skin thickness $t_{s}$ for 3D printing, (b) the folded compact configuration.

The angle $\alpha$ measured between the adjacent mountain and valley creases is $45^{\circ}$, which allows the dihedral angle $\varphi_{i 3}$ has a rotation range of $\left[0,180^{\circ}\right]$.

To make one module of the four-sided accordion fold pattern, at least four rectangular tile bases are required and jointed in a sequence as shown in Fig. 4. The kinematic equivalent of this assembly is a mechanism consists of four identical 1-DOF double- 
centered 6R linkages jointed to each other with rotary hinges, of which the axes are perpendicular to the collinear joint axes passing two spherical centers. Taking all the panels as rigid links, the mechanism is also a 1-DOF system during the folding process. When the dihedral angle $\varphi_{i 3}$ change from $0^{\circ}$ of the configuration in Fig. 4(a) to $180^{\circ}$, the mechanism reconfigures to a compact configuration in Fig. 4(b). In this flat-folded configuration, the height of the assembly is purely related to the thickness of the panels and the membrane.

\section{Fabrication of the Deployable Module: Skeleton 3D Printing and Membrane Adhesion}

With advancement of multi-material additive-manufacturing technologies particularly the $3 \mathrm{D}$ printing using soft materials, a number of cost effective approaches have been developed for making both rigid and soft robotic systems [26,27]. In this section, we presents the 3D printed origami skeleton using TPU filament and the fabrication of the deployable module by adhering the foldable skeleton to thin sheet TPU coated fabric.

(a)

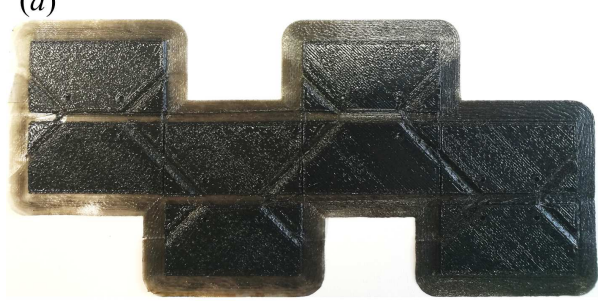

(b)

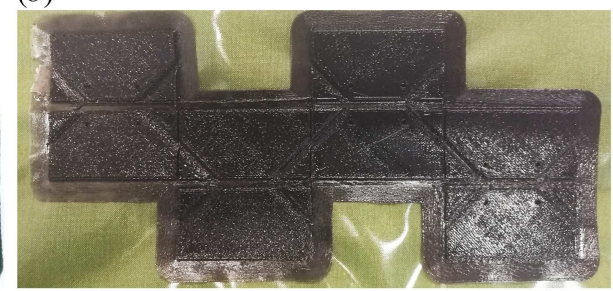

Fig. 5. Fabrication of the deployable module (a) 3D printed foldable skeleton using TPU 95A (black), (b) the laminated material in which the skeleton is adhered to the TPU coated fabric (green).

We use a commercial 3D printer, the Ultimaker 3 , with dual $0.4 \mathrm{~mm}$ nozzles to print the skeleton structure. The printable filaments including Nylon, ABS, PLA and TPU 95A are considered while all data sheets of materials show that TPU 95A is the only material with flexibility and resistant to wear and tear. The selection of materials for the membrane skin is concurrently considered together with materials for printing origami skeleton. Considering both mechanical properties and compatibility with other materials, a type of air tight TPU coated fabric is selected for the membrane skin.

With the flat sheet model illustrated in Fig. 4, samples of foldable skeleton (Fig.5) were printed using Ultimaker TPU 95A. In the printing setting, the adhesion layer is particularly used to maintain the gap assigned to mountain and valley creases thus the geometry of the pattern.

The 3D printed skeleton is then laminated to the TPU coated fabric using a steam iron for preliminary validation of the fabrication process. The welding temperature for laminating the skeleton and the TPU fabric is tested between $120^{\circ} \mathrm{C}$ and $200^{\circ} \mathrm{C}$. To 
achieve best adhesion of the two components, the maximum temperature was used for the materials selected in this paper. Due to the high temperature, the TPU fabric was wrinkled slightly at the area without skeleton (Fig. 5(b)). The final step of the fabrication

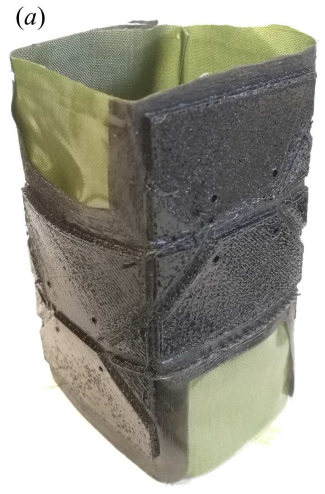

(b)



(c)

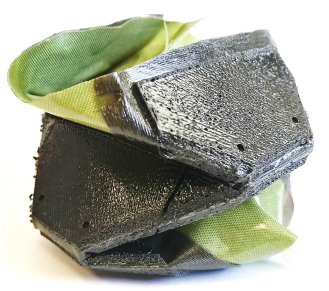

Fig. 6. The prototype of the deployable module. (a) the fully deployed configuration, (b) the partially folded configuration, (c) the folded configuration (not flat due to the compliance of the fabric).

process is to seal the open creases thus form a four-sided accordion structure made of TPU fabric with 3D printed origami skeleton. As illustrated in Fig. 6(a), the module is in its fully deployed configuration due to the stiffness of the TPU fabric. Apply external force along the axis of the module, it can be passively folded into a partially folded configuration (Fig. 6(b)) and finally the near compact configuration (Fig. 6(c)). It needs to mention that the deformation that occurs in the panels of the compliant models and small motions at the flexible hinges make the module deployable.

\section{Conclusions}

This paper presented an engineering design process for developing foldable origami based deployable modules of robotic devices with 3D printed origami skeletons. The proposed process concurrently combines conceptual design, detailed design, materials selection and 3D printing and thermal laminate based fabrication phases. Based on the design process, a modified accordion fold pattern is adapted in the development of a lightweight deployable module, which consists of 3D printing origami skeleton and thermoplastic polyurethane (TPU) coated fabric as flexible membrane. The preliminary inspection proves that the 3D printed foldable skeleton enables controllable motion of the deployable module as the foldable motion and compliance are enabled mainly by the flexible membrane between the gaps of of origami skeletons panels. The proposed design and fabrication approach is beneficial to scalability of size thus adaptable to different working conditions. The combination of technologies including 3D printing 
using soft filament and thermal lamination provides a cost effective approach for making deployable soft devices based on additive manufacturing. Future work will focus on developed of a complete robotic arm a number of the deployable modules, integration of actuation system and motion control.

\section{References}

1. Kanade, T.: A theory of origami world. Artificial intelligence 13(3), 279-311 (1980)

2. Lang, R.J.: Origami design secrets: mathematical methods for an ancient art. AK Peters/CRC Press (2011)

3. Dai, J.S., Jones, J.R.: Kinematics and mobility analysis of carton folds in packing manipulation based on the mechanism equivalent. Proceedings of the Institution of Mechanical Engineers, Part C: Journal of Mechanical Engineering Science 216(10), 959-970 (2002)

4. Howell, L.L.: Compliant mechanisms. John Wiley \& Sons (2001)

5. Greenberg, H., Gong, M.L., Magleby, S.P., Howell, L.L.: Identifying links between origami and compliant mechanisms. Mechanical Sciences 2(2), 217-225 (2011)

6. Song, J., Chen, Y., Lu, G.: Axial crushing of thin-walled structures with origami patterns. Thin-Walled Structures 54, 65-71 (2012)

7. Ma, J., You, Z.: Energy absorption of thin-walled square tubes with a prefolded origami patternpart i: geometry and numerical simulation. Journal of Applied Mechanics 81(1), 011003 (2014)

8. Martinez, R.V., Fish, C.R., Chen, X., Whitesides, G.M.: Elastomeric origami: programmable paper-elastomer composites as pneumatic actuators. Advanced functional materials 22(7), 1376-1384 (2012)

9. An, S.M., Ryu, J., Cho, M., Cho, K.J.: Engineering design framework for a shape memory alloy coil spring actuator using a static two-state model. Smart Materials and Structures 21(5), 055009 (2012)

10. Zhang, K., Qiu, C., Dai, J.S.: An extensible continuum robot with integrated origami parallel modules. Journal of Mechanisms and Robotics 8(3), 031010 (2016)

11. Felton, S., Tolley, M., Demaine, E., Rus, D., Wood, R.: A method for building self-folding machines. Science 345(6197), 644-646 (2014)

12. Miyashita, S., Guitron, S., Ludersdorfer, M., Sung, C.R., Rus, D.: An untethered miniature origami robot that self-folds, walks, swims, and degrades. In: 2015 IEEE International Conference on Robotics and Automation (ICRA). pp. 1490-1496. IEEE (2015)

13. Salerno, M., Zhang, K., Menciassi, A., Dai, J.S.: A novel 4-dof origami grasper with an sma-actuation system for minimally invasive surgery. IEEE Transactions on Robotics 32(3), 484-498 (2016)

14. Kim, S.J., Lee, D.Y., Jung, G.P., Cho, K.J.: An origami-inspired, self-locking robotic arm that can be folded flat. Science Robotics 3(16), eaar2915 (2018)

15. Zhakypov, Z., Paik, J.: Design methodology for constructing multimaterial origami robots and machines. IEEE Transactions on Robotics 34(1), 151-165 (2018)

16. Liu, T., Wang, Y., Lee, K.: Three-dimensional printable origami twisted tower: Design, fabrication, and robot embodiment. IEEE Robotics and Automation Letters 3(1), 116-123 (2018)

17. Li, S., Vogt, D.M., Rus, D., Wood, R.J.: Fluid-driven origami-inspired artificial muscles. Proceedings of the National Academy of Sciences 114(50), 13132-13137 (2017). https://doi.org/10.1073/pnas.1713450114

18. Zhang, K., Zhu, Y., Lou, C., Zheng, P., Kovač, M.: A design and fabrication approach for pneumatic soft robotic arms using 3d printed origami skeletons. In: The 2019 IEEE International Conference on Soft Robotics (RoboSoft 2019). pp. 1-7. IEEE (2019) 
19. Guest, S.D., Pellegrino, S.: The folding of triangulated cylinders, part i: geometric considerations. Journal of applied mechanics 61(4), 773-777 (1994)

20. Morgan, J., Magleby, S.P., Howell, L.L.: An approach to designing origami-adapted aerospace mechanisms. Journal of Mechanical Design 138(5), 052301 (2016)

21. Tachi, T., Miura, K.: Rigid-foldable cylinders and cells. Journal of the international association for shell and spatial structures 53(4), 217-226 (2012)

22. Zirbel, S.A., Lang, R.J., Thomson, M.W., Sigel, D.A., Walkemeyer, P.E., Trease, B.P., Magleby, S.P., Howell, L.L.: Accommodating thickness in origami-based deployable arrays. Journal of Mechanical Design 135(11), 111005 (2013)

23. Zhang, K., Dai, J.S.: Classification of origami-enabled foldable linkages and emerging applications. In: ASME 2013 International Design Engineering Technical Conferences and Computers and Information in Engineering Conference. pp. V06BT07A024-V06BT07A024. American Society of Mechanical Engineers (2013)

24. Chen, Y., Feng, H., Ma, J., Peng, R., You, Z.: Symmetric waterbomb origami. Proceedings of the Royal Society A: Mathematical, Physical and Engineering Sciences 472(2190), 20150846 (2016)

25. Chen, Y., Peng, R., You, Z.: Origami of thick panels. Science 349(6246), 396-400 (2015)

26. MacCurdy, R., Katzschmann, R., Kim, Y., Rus, D.: Printable hydraulics: A method for fabricating robots by 3d co-printing solids and liquids pp. 3878-3885 (May 2016). https://doi.org/10.1109/ICRA.2016.7487576

27. Zhang, K., Chermprayong, P., Tzoumanikas, D., Li, W., Grimm, M., Smentoch, M., Leutenegger, S., Kovac, M.: Bioinspired design of a landing system with soft shock absorbers for autonomous aerial robots. Journal of Field Robotics $\mathbf{0}(0)$. https://doi.org/10.1002/rob.21840 